\title{
Analysis of capacitive force acting on a cantilever tip at solid/liquid interfaces
}

\section{$\operatorname{AUTHOR}(S)$ :}

Umeda, Ken-ichi; Kobayashi, Kei; Oyabu, Noriaki; Hirata, Yoshiki; Matsushige, Kazumi; Yamada, Hirofumi

\section{CITATION:}

Umeda, Ken-ichi ...[et al]. Analysis of capacitive force acting on a cantilever tip at solid/liquid interfaces. Journal of Applied Physics 2013, 113(15): 154311.

\section{ISSUE DATE:}

2013-04-18

URL:

http://hdl.handle.net/2433/187962

\section{RIGHT:}

(c) 2013 American Institute of Physics. This article may be downloaded for personal use only. Any other use requires prior permission of the author and the American Institute of Physics. 


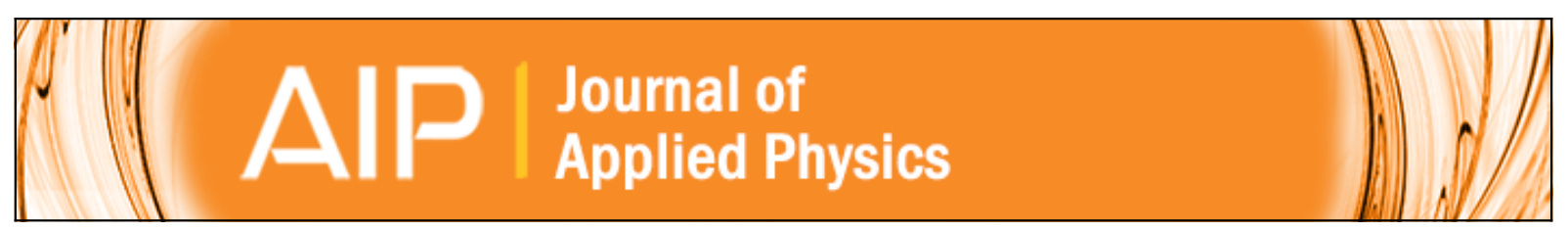

\section{Analysis of capacitive force acting on a cantilever tip at solid/liquid interfaces}

Ken-ichi Umeda, Kei Kobayashi, Noriaki Oyabu, Yoshiki Hirata, Kazumi Matsushige, and Hirofumi Yamada

Citation: Journal of Applied Physics 113, 154311 (2013); doi: 10.1063/1.4801795

View online: http://dx.doi.org/10.1063/1.4801795

View Table of Contents: http://scitation.aip.org/content/aip/journal/jap/113/15?ver=pdfcov

Published by the AIP Publishing

\section{Articles you may be interested in}

Analysis of a static undulation on the surface of a thin dielectric liquid layer formed by dielectrophoresis forces J. Appl. Phys. 110, 024107 (2011); 10.1063/1.3606435

Mechanical to electrical energy conversion in a hybrid liquid-solid dielectric electrostatic generator J. Appl. Phys. 106, 044108 (2009); 10.1063/1.3207834

Two- and four-electrode, wide-bandwidth, dielectric spectrometer for conductive liquids: Theory, limitations, and experiment

J. Appl. Phys. 102, 104106 (2007); 10.1063/1.2815666

Dielectric behavior of polymer-stabilized-liquid-crystal cells made from hosts with different ultraviolet absorptions J. Appl. Phys. 96, 4366 (2004); 10.1063/1.1790589

Current, charge, and capacitance during scanning probe oxidation of silicon. II.Electrostatic and meniscus forces acting on cantilever bending

J. Appl. Phys. 96, 2393 (2004); 10.1063/1.1771821

\section{AlP Re-register for Table of Content Alerts}




\title{
Analysis of capacitive force acting on a cantilever tip at solid/liquid interfaces
}

\author{
Ken-ichi Umeda, ${ }^{1}$ Kei Kobayashi, ${ }^{2}$ Noriaki Oyabu, ${ }^{1,3}$ Yoshiki Hirata, ${ }^{4}$ Kazumi Matsushige, ${ }^{1}$ \\ and Hirofumi Yamada ${ }^{1, a)}$ \\ ${ }^{1}$ Department of Electronic Science and Engineering, Kyoto University, Katsura, Nishikyo, \\ Kyoto 615-8510, Japan \\ ${ }^{2}$ Office of Society-Academia Collaboration for Innovation, Kyoto University, Katsura, Nishikyo, \\ Kyoto 615-8520, Japan \\ ${ }^{3}$ JST Development of Systems and Technology for Advanced Measurement and Analysis, Honcho, \\ Kawaguchi 332-0012, Japan \\ ${ }^{4}$ Institute for Biological Resources and Functions, National Institute of Advanced Industrial \\ Science and Technology, 1-1-1 Higashi, Tsukuba 305-8566, Japan
}

(Received 6 March 2013; accepted 28 March 2013; published online 18 April 2013)

\begin{abstract}
Dielectric properties of biomolecules or biomembranes are directly related to their structures and biological activities. Capacitance force microscopy based on the cantilever deflection detection is a useful scanning probe technique that can map local dielectric constant. Here we report measurements and analysis of the capacitive force acting on a cantilever tip at solid/liquid interfaces induced by application of an alternating voltage to explore the feasibility of the measurements of local dielectric constant by the voltage modulation technique in aqueous solutions. The results presented here suggest that the local dielectric constant measurements by the conventional voltage modulation technique are basically possible even in polar liquid media. However, the cantilever deflection is not only induced by the electrostatic force, but also by the surface stress, which does not include the local dielectric information. Moreover, since the voltage applied between the tip and sample are divided by the electric double layer and the bulk polar liquid, the capacitive force acting on the apex of the tip are strongly attenuated. For these reasons, the lateral resolution in the local dielectric constant measurements is expected to be deteriorated in polar liquid media depending on the magnitude of dielectric response. Finally, we present the criteria for local dielectric constant measurements with a high lateral resolution in polar liquid media. (C) 2013 AIP Publishing LLC [http://dx.doi.org/10.1063/1.4801795]
\end{abstract}

\section{INTRODUCTION}

Recently, significant progress has been made in dynamicmode atomic force microscopy (AFM) operating in liquid environments, ${ }^{1,2}$ which opened a new way to directly investigate "in vivo" biological processes ${ }^{1-4}$ as well as redox reaction dynamics of various molecules ${ }^{5,6}$ For further applications of dynamic-mode AFM, especially for biological and electrochemical applications, development of a method for investigating local surface charge or potential distribution is strongly required. Investigation of dielectric properties of biomolecules in physiological environments is essentially important for understanding dynamics of the biomolecules. ${ }^{7,8}$ However, since the surface charges are screened by the surrounding counter ions in aqueous solutions, forming an electric double layer (EDL), the electrostatic interaction between the AFM tip and the sample surface is not as simple as that in vacuum environments or in the ambient conditions.

Electrostatic force microscopy (EFM), ${ }^{9,10}$ scanning capacitance microscopy (SCM), ${ }^{1-13}$ and scanning impedance microscopy (SIM) ${ }^{14,15}$ which are the scanning probe techniques based on AFM, are used for dielectric constant measurement with nanometer-scale resolution. These methods have been commonly used either in vacuum environments or

${ }^{a)}$ E-mail: h-yamada@kuee.kyoto-u.ac.jp. in the ambient conditions. In particular, the EFM has been applied to wide variety of samples, such as dielectric nanoparticles, inorganic/organic sheets, carbon nanotubes, biomembranes etc. ${ }^{16-19}$ This method is based on the detection of the electrostatic force induced by an alternating voltage applied between the tip and sample surface. In fact, the EFM is possibly applied to liquid environment because the parasitic capacitance originating from the other parts than the tip and the cantilever does not need to be considered. ${ }^{20}$ On the other hand, although there have been some investigations on the electrostatic force acting on the AFM cantilever with a tip when the alternating voltage is applied between the cantilever tip and sample in liquid media, ${ }^{7,21-26}$ no systematic study has been conducted on this topic to the authors' knowledge.

Recently, we discussed the feasibility to measure local surface charge or potential distribution at solid/liquid interfaces by the voltage modulation techniques in aqueous solutions. ${ }^{27}$ In this paper, we report measurements and analysis of the capacitive force acting on a cantilever with a tip induced by the alternating voltage application in various liquid media. We measured the dependence of the capacitive force on the frequency of the alternating voltage and on the tip-sample distance. The experimental results are compared with theoretical calculations for exploring the feasibility at the measurements of local dielectric properties at solid/liquid 
interfaces by the voltage modulation technique in aqueous solutions.

The paper is organized as follows. After a brief description of the experimental conditions in Sec. II, we discuss the dependence of the capacitive force on the frequency of the alternating voltage and on the distance between a cantilever with a tip and a sample in various liquid media in Secs. III and IV, respectively. In Sec. V, a numerical analysis of the capacitive force in polar liquid media is given. Finally, in Sec. VI, we discuss the criteria for local surface charge, potential and dielectric constant measurements in aqueous solutions.

\section{MATERIAL AND METHODS}

\section{A. Instruments}

Figure 1 shows a schematic illustration of an experimental setup. We used a customized AFM instrument (Shimadzu: SPM-9600) with a home-built digital controller programmed using LabVIEW (National Instruments). We used a rectangular cantilever with platinum iridium coatings on both sides (Nanosensors: PPP-NCSTPt), whose spring constant $\left(k_{z}\right)$ was $5.3 \mathrm{~N} / \mathrm{m}$, calibrated using Sader's method in air. ${ }^{28}$ The thermal deflection noise density spectrum was measured in each medium, and it was fitted to a damped harmonic oscillator model to calibrate the sensitivity of the deflection sensor. We used a polycrystalline platinum plate as a sample. A lock-in amplifier with a signal source (AMETEK: 7280) was used to apply an alternating voltage of an angular frequency $\omega_{\mathrm{m}}$ between the cantilever tip and sample, and to detect the amplitude and phase of the fundamental or second harmonic component in the cantilever deflection signal.

\section{B. Liquid media}

Liquid media used in the experiments are classified into three categories: non-polar solvent, protic polar solvents, and aprotic polar solvent. A fluorocarbon liquid (3M: Fluorinert FC-70) was used as a non-polar and insulating solvent. $1 \mathrm{mM}$ $\mathrm{KCl}$ aqueous solution made by dissolving potassium chloride reagent (min. 99.5\%) (Wako Pure Chemical Industries) in

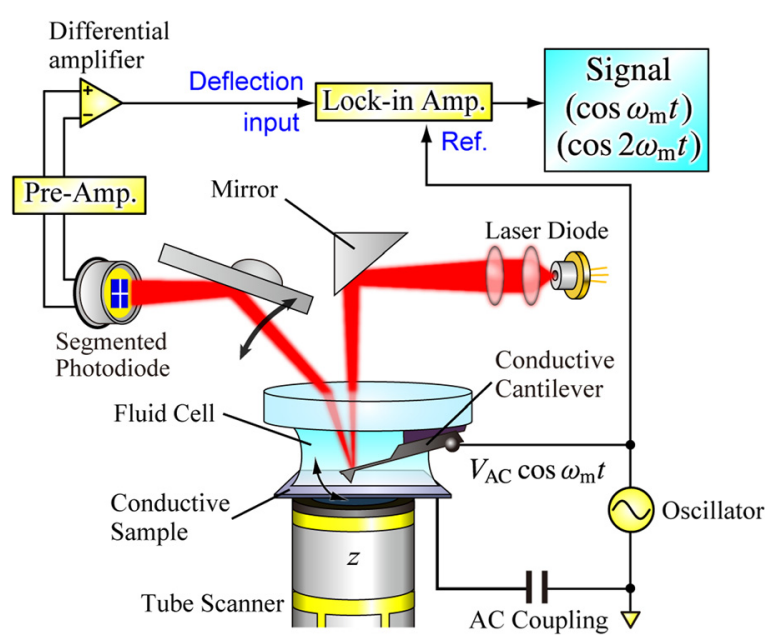

FIG. 1. Schematic illustration of experimental setup for detection of capacitive force acting on a conductive cantilever tip induced by applying an alternating voltage between the cantilever tip and a conductive sample surface.
TABLE I. Physical properties of the liquid media used in the experiments. ${ }^{29-31}$

\begin{tabular}{lccc}
\hline \hline Medium & $\begin{array}{c}\text { Relative } \\
\text { permittivity }\left(\varepsilon_{\mathrm{r}}\right)\end{array}$ & $\begin{array}{c}\text { Resistivity } \\
(\rho)[\Omega \mathrm{cm}]\end{array}$ & $\begin{array}{c}\text { Characteristic dielectric } \\
\text { relaxation frequency } \\
\left(f_{\mathrm{c}}\right)[\mathrm{Hz}]\end{array}$ \\
\hline${\mathrm{FC}-70^{20}}^{21}$ & 1.98 & $2.3 \times 10^{15}$ & - \\
Water $^{21}$ & 78.4 & $1.82 \times 10^{7}$ & $1.26 \mathrm{k}$ \\
$\mathrm{KCl}(1 \mathrm{mM})^{30,31}$ & 78.4 & $6.80 \times 10^{3}$ & $3.37 \mathrm{M}$ \\
Acetonitrile $^{21}$ & 37.5 & $1.67 \times 10^{9}$ & 28.8 \\
Acetic acid $^{21}$ & 6.15 & $1.67 \times 10^{8}$ & $1.75 \mathrm{k}$ \\
\hline \hline
\end{tabular}

ultrapure water (Millipore), and an acetic acid reagent (99\%) (Nacalai Tesque) were used as protic polar solvents. A dehydrated acetonitrile reagent $(99 \%)$ (Wako Pure Chemical Industries) is used as an aprotic polar solvent. Reagents were used as supplied by the manufacturers without further purification. Table I summarizes the physical properties of the liquid media.

\section{FREQUENCY RESPONSE OF CAPACITIVE FORCE IN VARIOUS MEDIA}

In this section, we discuss the frequency response curves of the capacitive force in various media including polar liquid media. The electrostatic force acting on the tip and sample in EFM or KFM $\left(F_{\text {esf }}\right)$ is generally described as

$$
F_{\mathrm{esf}}=\frac{1}{2} \frac{\partial C_{\mathrm{ts}}}{\partial z} V^{2}
$$

where $C_{\mathrm{ts}}$ is a capacitance between the tip and sample, and $z$ is the tip-sample distance. $V$ is an effective voltage, which is the sum of externally applied modulation voltage $V_{\text {mod }}$ and the surface potential difference of the tip and sample $V_{\mathrm{SP}}$. When a modulation voltage of an amplitude $V_{\mathrm{ac}}$ at the angular frequency $\omega_{\mathrm{m}}$ with a dc offset voltage $V_{\mathrm{dc}}$, given by $V_{\text {mod }}=V_{\mathrm{dc}}+V_{\mathrm{ac}} \cos \omega_{\mathrm{m}} t$, is applied between the tip and sample, the electrostatic force becomes ${ }^{32,33}$

$$
\begin{aligned}
F_{\mathrm{esf}}= & \frac{1}{2} \frac{\partial C_{\mathrm{ts}}}{\partial z}\left[\left(V_{\mathrm{dc}}+V_{\mathrm{SP}}\right)^{2}+\frac{1}{2} V_{\mathrm{ac}}^{2}+2\left(V_{\mathrm{dc}}+V_{\mathrm{SP}}\right) V_{\mathrm{ac}}\right. \\
& \left.\times \cos \omega_{\mathrm{m}} t+\frac{1}{2} V_{\mathrm{ac}}^{2} \cos 2 \omega_{\mathrm{m}} t\right]
\end{aligned}
$$

The third and fourth terms are referred to as $1 \omega_{\mathrm{m}}$ and $2 \omega_{\mathrm{m}}$ components, respectively, hereafter. The electrostatic force of $2 \omega_{\mathrm{m}}$ component, which contains dielectric information, is referred to as the capacitive force. In KFM, $V_{\mathrm{dc}}$ is controlled so that the $1 \omega_{\mathrm{m}}$ component is nulled, thereby $V_{\mathrm{SP}}$ can be measured as $V_{\mathrm{SP}}=-V_{\mathrm{dc}}$. However, in the case of polar media, the $1 \omega_{\mathrm{m}}$ component cannot be nulled by simply controlling $V_{\mathrm{dc}}$ thereby $V_{\mathrm{SP}}$ cannot be measured by the conventional KFM setup. ${ }^{27}$ On the other hand, the $2 \omega_{\mathrm{m}}$ component does not depend on $V_{\mathrm{dc}}$ but does depend on $V_{\mathrm{ac}}$ with a relatively high reproducibility. Here we compare the frequency response and the tip-sample distance dependence of the $2 \omega_{\mathrm{m}}$ component, the capacitive force, in various media to understand the 
(a)

(b)

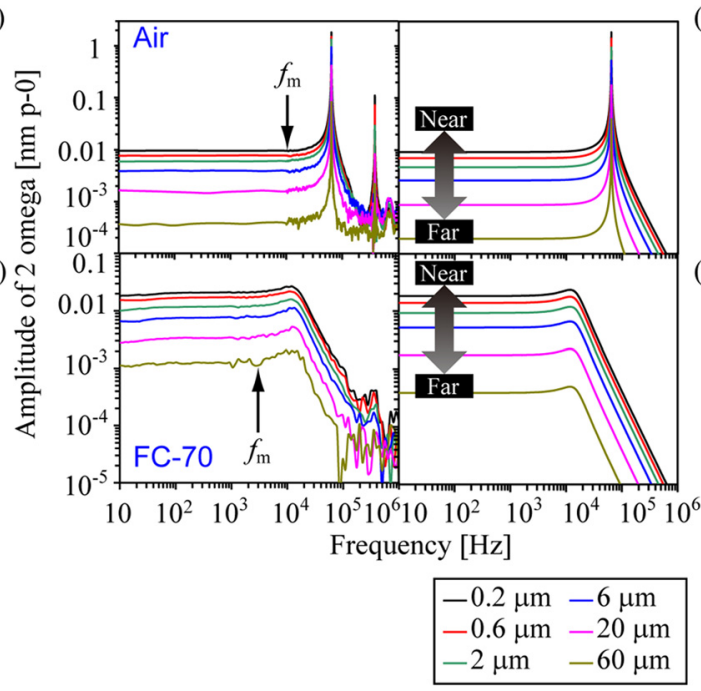

FIG. 2. Frequency response curves of amplitude of $2 \omega_{\mathrm{m}}$ component in the cantilever deflection signal as a function of $2 f_{\mathrm{m}}$ measured in (a) air and (b) fluorocarbon liquid, at various tip-sample distances. (c) and (d) are theoretically calculated frequency response curves. The arrows indicate the modulation frequencies used in the experiments in Sec. VI.

electrostatic force acting on the cantilever with a tip induced by the alternating voltage in polar liquid media.

Figures 2(a) and 2(b) show the frequency response curves of the amplitude of the capacitive force measured in air and fluorocarbon liquid at various tip-sample distances while $V_{\text {ac }}$ was fixed at $1.4 \mathrm{~V}$ peak-to-zero. The plots are shown as a function of $2 f_{\mathrm{m}}$. Figure 3 shows a schematic of a model of a cantilever with a conical tip for calculating the capacitive force acting on a rectangular cantilever with a tip. We divided the capacitive force acting on a rectangular cantilever with a tip into three parts, as described in Refs. 34 and 35 as

$$
F_{\mathrm{esf}}^{2 \omega_{\mathrm{m}}}=F_{\text {apex }}^{2 \omega_{\mathrm{m}}}+F_{\text {cone }}^{2 \omega_{\mathrm{m}}}+F_{\mathrm{cl}}^{2 \omega_{\mathrm{m}}}
$$

which are the capacitive force acting on a spherical tip apex, that acting on a conical part of the tip, and that acting on the cantilever, respectively. $F_{\text {apex }}^{2 \omega_{\mathrm{m}}}$ and $F_{\text {cone }}^{2 \omega_{\mathrm{m}}}$ are given after Ref. 34 (see Subsection 1 of Appendix). The equation for $F_{\mathrm{cl}}^{2 \omega_{\mathrm{m}}}$ was

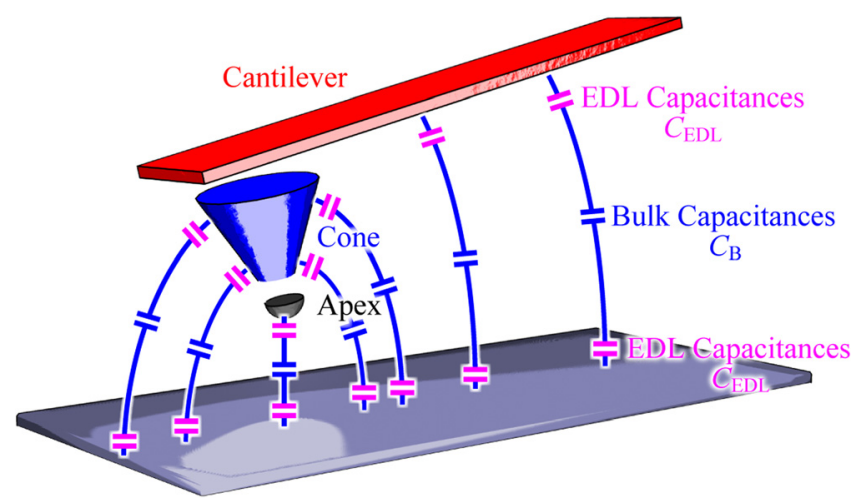

FIG. 3. Schematic of a model of a cantilever with a conical tip for calculating the electrostatic force acting on a cantilever with a tip in polar liquid media. The EDL capacitances at the tip side and the sample side are connected to the bulk solution capacitance in series. The components other than capacitances such as resistances are omitted for clarity. slightly modified from that given in Ref. 35 considering the non-uniform distribution of the capacitive force, ${ }^{36,37}$ and differentiating the capacitance along $z$ axis, and it is

$$
F_{\mathrm{cl}}^{2 \omega_{\mathrm{m}}}=\frac{1}{8} \varepsilon_{0} \varepsilon_{\mathrm{r}} V_{\mathrm{ac}}^{2} w \frac{\cot \left(\theta_{\mathrm{cl}} / 2\right)}{\theta_{\mathrm{cl}}} \int_{r_{0}}^{r_{0}+l} \frac{\phi_{1}\left(l+r_{0}-x\right)}{\phi_{1}(l)} \frac{1}{x^{2}} \mathrm{~d} x
$$

where $\varepsilon_{0}$ and $\varepsilon_{\mathrm{r}}$ are the dielectric constant of vacuum and relative dielectric constant, and $l, w$ and $\theta_{\mathrm{cl}}$ are the length, width, and tilt angle of the cantilever. $r_{0}$ represents the location of the cantilever end, which is given by

$$
r_{0}=\frac{z+h}{2} \cot \left(\frac{\theta_{\mathrm{cl}}}{2}\right) \text {. }
$$

$\phi_{1}$ is the eigenfunction of the first eigenmode given by

$$
\begin{aligned}
\phi_{1}(x)= & \left(\cos k_{1} x-\cosh k_{1} x\right) \\
& -\frac{\cos k_{1} l+\cosh k_{1} l}{\sin k_{1} l+\sinh k_{1} l}\left(\sin k_{1} x-\sinh k_{1} x\right),
\end{aligned}
$$

where $k_{1} l$ is the first eigenvalue (1.8751). The complex amplitude of the cantilever at $2 \omega_{\mathrm{m}}\left(A^{2 \omega_{\mathrm{m}}}\right)$, induced by $F_{\mathrm{esf}}^{2 \omega_{\mathrm{m}}}$, is expressed by

$$
A^{2 \omega_{\mathrm{m}}}=G_{\mathrm{cl}} F_{\mathrm{esf}}^{2 \omega_{\mathrm{m}}}
$$

where the transfer function of the cantilever $G_{\mathrm{cl}}$ as a damped oscillator model

$$
G_{\mathrm{cl}}=\frac{Q_{1}}{Q_{1}\left[1-\left(f_{\mathrm{m}} / f_{1}\right)^{2}\right]+\mathrm{i}\left(f_{\mathrm{m}} / f_{1}\right)} \frac{1}{k_{z}},
$$

where $f_{1}$ and $Q_{1}$ are the resonance frequency and the mechanical quality factor of the first eigenmode. Figures 2(c) and 2(d) are theoretically calculated frequency response curves of the amplitude of the capacitive force in air and fluorocarbon liquid. The fitting parameters used for the calculations are summarized in Table II. The parameters of a model of a cantilever with a conical tip are also given in Table III. The dimensions of the cantilever were taken from the datasheet.

Figures 4(a)-4(c) compare the frequency response curves of the amplitude of the capacitive force in polar liquid media, which are $1 \mathrm{mM} \mathrm{KCl}$ aqueous solution, acetonitrile, and acetic acid, respectively. As we previously reported in Ref. 24, the cantilever deflection was induced by the surface stress, ${ }^{38}$ especially in the low frequency range, as well as by the electrostatic force in the high frequency range. Here,

TABLE II. Fitting parameters for theoretical frequency response curves in Figs. 2 and 4.

\begin{tabular}{lcccc}
\hline \hline Medium & $Q_{1}$ & $f_{1} / 2(\mathrm{kHz})$ & $\left|F_{\text {sso }}\right|(\mathrm{nN}$ peak-to-zero $)$ & $f_{\text {ss_c }} / 2(\mathrm{~Hz})$ \\
\hline Air & 220 & 64.1 & - & - \\
FC-70 & 1.15 & 14.9 & - & - \\
KCl $(1 \mathrm{mM})$ & 4.3 & 25.9 & 79.5 & 150 \\
Acetonitrile & 5.0 & 30.3 & 9.01 & $\sim 30$ \\
Acetic acid & 3.0 & 22.3 & 2.65 & $\sim 3$ \\
\hline \hline
\end{tabular}


TABLE III. Parameters of a model of a cantilever with a tip.

\begin{tabular}{lc}
\hline \hline Parameter & Value \\
\hline Spring constant $\left(k_{z}\right)$ & $5.3 \mathrm{~N} / \mathrm{m}$ \\
Radius of curvature of tip apex $\left(R_{\text {apex }}\right)$ & $20 \mathrm{~nm}$ \\
Half cone angle $\left(\theta_{\text {cone }}\right)$ & $20^{\circ}$ \\
Width of cantilever $(w)$ & $22 \mu \mathrm{m}$ \\
Length of cantilever $(l)$ & $152 \mu \mathrm{m}$ \\
Tip height $(h)$ & $10.9 \mu \mathrm{m}$ \\
Tilt angle of cantilever $\left(\theta_{\mathrm{cl}}\right)$ & $12^{\circ}$ \\
Poisson's ratio $(\nu)$ & 0.28 \\
\hline \hline
\end{tabular}

$A^{2 \omega_{\mathrm{m}}}$ is modeled as the sum of the contribution of $F_{\mathrm{esf}}^{2 \omega_{\mathrm{m}}}$ and surface stress $F_{\mathrm{ss}}^{2 \omega_{\mathrm{m}}}$ as expressed by

$$
\begin{aligned}
A^{2 \omega_{\mathrm{m}}} & =G_{\mathrm{cl}}\left(F_{\mathrm{ss}}^{2 \omega_{\mathrm{m}}}+F_{\mathrm{esf}}^{2 \omega_{\mathrm{m}}}\right) \\
& =G_{\mathrm{cl}}\left[\frac{1}{1+\mathrm{i}\left(f / f_{\mathrm{ss} \_\mathrm{c}}\right)^{\alpha}} F_{\mathrm{ss} 0}+F_{\mathrm{esf}}^{2 \omega_{\mathrm{m}}}\right],
\end{aligned}
$$

where $F_{\text {ss0 }}$ and $f_{\text {ss_c }}$ are fitting parameters, a complex effective surface stress force $\left(\left|F_{\mathrm{ss} 0}\right| \mathrm{e}^{-\mathrm{i} \theta_{\mathrm{ss} 0}}\right)$ at a low-frequency limit and a cutoff frequency, respectively. ${ }^{24}$ The cutoff frequency of the surface stress is equal to the cutoff frequency of a series circuit composed of the bulk solution resistance and the electric double layer capacitance (see Subsection 3 of Appendix). The parameter $\alpha$ usually ranges from 0.5 to 1.0 because both electrodes are typically not in ideal conditions. ${ }^{39}$ The solid curves in Figs. 4(d)-4(f) are theoretically calculated frequency response curves taking both the electrostatic force and surface stress contribution into account. The

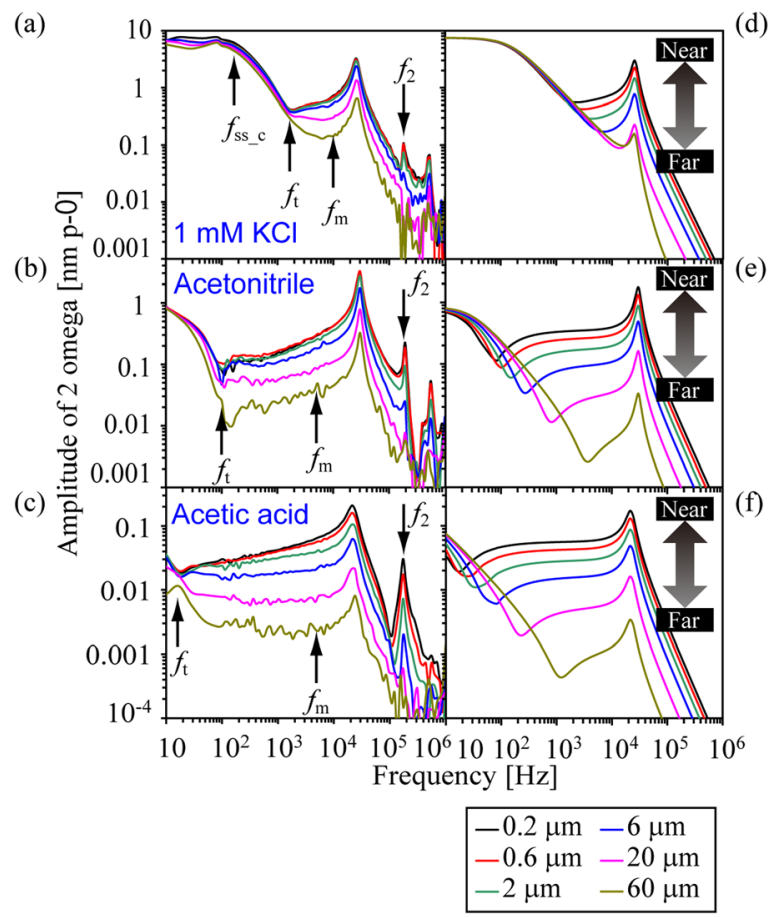

FIG. 4. Frequency response curves of amplitude of $2 \omega_{\mathrm{m}}$ component in the cantilever deflection signal as a function of $2 f_{\mathrm{m}}$ measured in (a) $1 \mathrm{mM} \mathrm{KCl}$ aqueous solution, (b) acetonitrile, and (c) acetic acid, at various tip-sample distances. (d)-(f) are theoretically calculated frequency response curves. phase delays of surface stress $\left(\theta_{\mathrm{ss} 0}\right)$ were 40,70 , and 60 degrees in $1 \mathrm{mM} \mathrm{KCl}$ aqueous solution, acetonitrile, and acetic acid, respectively. The contribution of the surface stress in the cantilever deflection becomes significant as the dielectric constant of the medium becomes larger. The transition frequency $\left(f_{\mathrm{t}}\right)$ is obviously dependent on the media. The lower the dielectric constant of the medium, the lower $f_{\mathrm{t}}$ becomes, and this can be explained by the equivalent circuit shown in Ref. 27. Since the surface stress is caused by the voltage applied to $C_{\mathrm{EDL}}, f_{\mathrm{t}}$ is determined by the bulk solution impedance. The best fitted values of $\left|F_{\text {sso }}\right|$ and $f_{\text {ss_c }}$ are shown in Table II. The electric double layer capacitance per $\delta C_{\mathrm{EDL}}$ calculated from $\left|F_{\mathrm{ss} 0}\right|$ are $1.45,0.27$, and $0.19 \mathrm{~F} / \mathrm{m}^{2}$ in $1 \mathrm{mM}$ $\mathrm{KCl}$ aqueous solution, acetonitrile, and acetic acid, respectively. $\delta C_{\mathrm{EDL}}$ calculated from $f_{\mathrm{ss} \_\mathrm{c}}$ in $1 \mathrm{mM} \mathrm{KCl}$ aqueous solution was 1.30 , which is almost same as the value calculated from $\left|F_{\mathrm{ss} 0}\right|$. On the other hand, $\delta C_{\mathrm{EDL}}$ calculated from $f_{\text {ss_c }}$ in acetonitrile and acetic acid were much lower than the values calculated from $\left|F_{\text {sso }}\right|$ because pure solvents contain some impurities of the order of a few $\mu \mathrm{M}$, which brings the resistivity lower.

In all media, the electrostatic force contribution in the frequency response curves agreed with the theoretically calculated curves. The frequency response curves in the range of higher than $f_{\mathrm{t}}$ recorded in the tip-sample distance ranging from 0.2 to $60 \mu \mathrm{m}$ were successfully reproduced by the calculations. In conventional EFM and KFM in vacuum or the ambient conditions, $f_{\mathrm{m}}$ is typically chosen at a frequency well below the resonance frequency ${ }^{32,33}$ or at the second resonance frequency. ${ }^{40}$ In the following section, the tip-sample dependence of the capacitive force in the frequency ranges of those practically chosen for EFM and KFM is analyzed.

\section{TIP-SAMPLE DISTANCE DEPENDENCE OF CAPACITIVE FORCE IN VARIOUS MEDIA}

Figure 5 shows the tip-sample distance dependence of the amplitude of the capacitive force in non-polar media, air and fluorocarbon liquid. $f_{\mathrm{m}}$ were chosen at $10 \mathrm{kHz}$, which was well below $f_{1}$. The amplitude increased when the tipsample distance was reduced to less than $100 \mathrm{~nm}$ both in air and in fluorocarbon liquid. Theoretically calculated curves for $F_{\mathrm{esf}}^{2 \omega_{\mathrm{m}}}$ agreed quantitatively with the measured curves with a slight deviation, which might be due to some errors in the cantilever parameters given in Table III. The electrostatic forces acting on the three parts, $F_{\text {apex }}^{2 \omega_{\mathrm{m}}}, F_{\text {cone }}^{2 \omega_{\mathrm{m}}}$, and $F_{\mathrm{cl}}^{2 \omega_{\mathrm{m}}}$ were also plotted in the Fig. 5.

Next, we compare the tip-sample distance dependences of the amplitude of the capacitive force in polar liquid media. Figures 6(a)-6(c) show the results obtained in $1 \mathrm{mM}$ $\mathrm{KCl}$ aqueous solution, acetonitrile, and acetic acid, respectively. $f_{\mathrm{m}}$ was chosen at $10 \mathrm{kHz}$, which was well below $f_{1}$. We did not observe the increase in the amplitude when the tip-sample distance was reduced. The experimentally measured curves agreed well with the theoretically calculated curves for $F_{\mathrm{cl}}^{2 \omega_{\mathrm{m}}}$, which suggests that $F_{\text {apex }}^{2 \omega_{\mathrm{m}}}$ and $F_{\text {cone }}^{2 \omega_{\mathrm{m}}}$ were negligibly small. This is because the EDL capacitances $\left(C_{\mathrm{EDL}}\right)$ are connected in series to the bulk solution capacitance $\left(C_{\mathrm{B}}\right)$ in polar liquid media as discussed in Ref. 27. The 
(a)

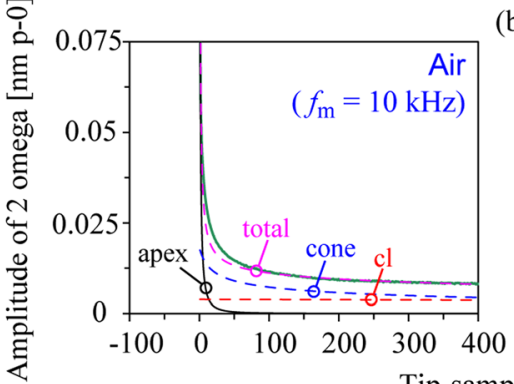

(b)

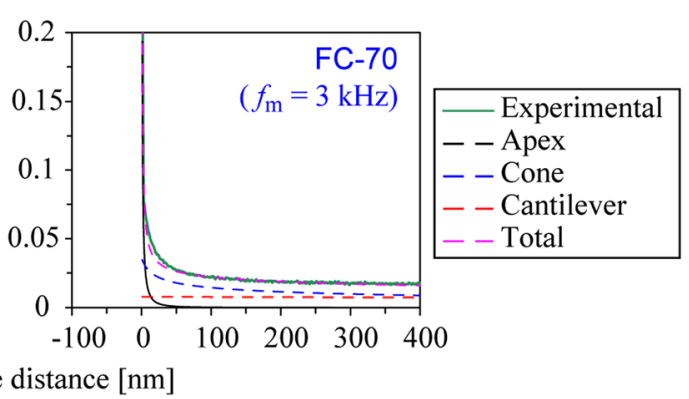

FIG. 5. Tip-sample distance dependence of amplitude of $2 \omega_{\mathrm{m}}$ component in the cantilever deflection signal measured when $f_{\mathrm{m}}$ were chosen at frequencies well below $f_{1}$ in (a) air and (b) fluorocarbon liquid. The solid curves show experimentally measured curves. The broken curves are theoretically calculated curves of the capacitive force acting on the tip apex $\left(F_{\text {apex }}^{2 \omega_{\mathrm{m}}}\right)$, the conical part of the tip $\left(F_{\text {cone }}^{2 \omega_{\mathrm{m}}}\right)$, the cantilever $\left(F_{\mathrm{cl}}^{2 \omega_{\mathrm{m}}}\right)$, and the total sum of all $\left(F_{\mathrm{esf}}^{2 \omega_{\mathrm{m}}}\right)$.

electrostatic force acting on the tip apex and conical part of the tip are attenuated when the applied voltage is effectively applied to $C_{\mathrm{EDL}}$ which are smaller than $C_{\mathrm{B}}$.

Since $f_{\mathrm{m}}$ is often tuned at the second resonance frequency $\left(f_{2}\right)^{40}$ in EFM and KFM, we also measured the tip-sample distance dependence of the amplitude of the capacitive force by tuning $f_{\mathrm{m}}$ at $f_{2}$ in polar liquid media. Not only an enhancement in the amplitude by the second resonance, the reduction of the surface stress contribution is expected by the increase of $f_{\mathrm{m}}$. Figures $6(\mathrm{~d})-6(\mathrm{f})$ show the results obtained in $1 \mathrm{mM} \mathrm{KCl}$ aqueous solution, acetonitrile, and acetic acid, respectively. In $1 \mathrm{mM} \mathrm{KCl}$ aqueous solution, the amplitude did not show any increase in Fig. 6(d), as the same as in Fig. 6(a). However, on the other hand, in acetonitrile and acetic acid, we observed the increases in the amplitude as the tip was brought closer to the surface, as shown in
Figs. 6(e) and 6(f), suggesting that there were some contribution from $F_{\text {apex }}^{2 \omega_{\mathrm{m}}}$ and $F_{\text {cone }}^{2 \omega_{\mathrm{m}}}$. The $f_{\mathrm{c}}$ of $1 \mathrm{mM} \mathrm{KCl}$ aqueous solution is higher than $f_{2}$, but $f_{\mathrm{c}}$ of acetonitrile and acetic acid are much lower than $f_{2}$ as listed in Table $\mathrm{I}$; therefore, $C_{\mathrm{D}}$ can be ignored in the case of acetonitrile and acetic acid. However, typical $C_{\mathrm{S}}$ value is small enough to screen the electrostatic force acting on the apex of the tip. These results suggest that we cannot detect the electrostatic force acting on the tip apex by using conventional voltage modulation techniques in polar liquid media.

\section{DEPENDENCES OF CAPACITIVE FORCES ON POLARIZATION OF MEDIA}

In this section, we calculate the capacitive force in polar liquid media to discuss the reason why we did not observe (a)

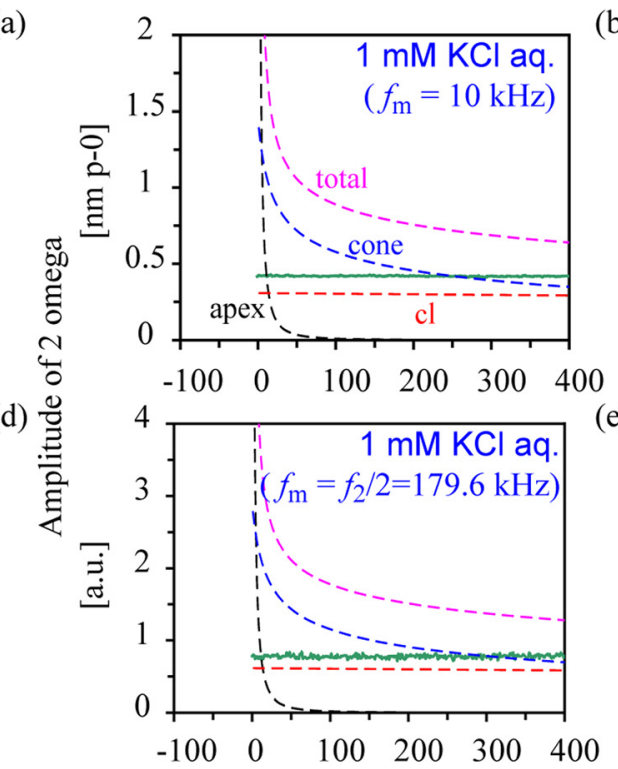

(b)

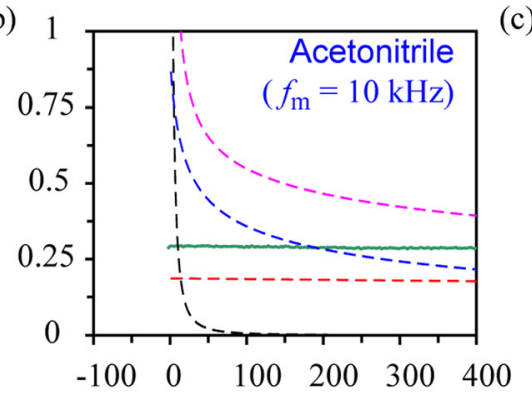

(c)

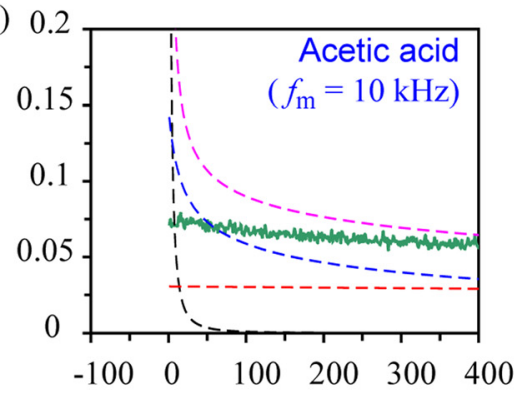

(e)

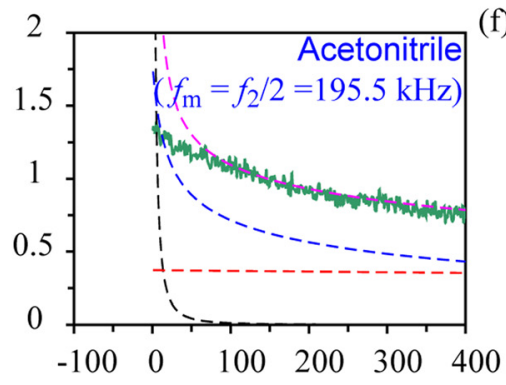

Tip-sample distance [nm] (f) 0.

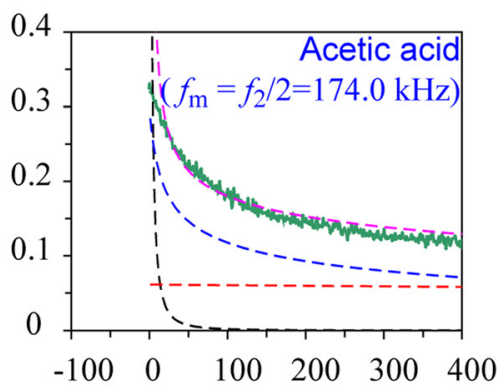

\begin{tabular}{|l|}
\hline - Experimental \\
- Apex \\
-- Cone \\
-- Cantilever \\
- - Total
\end{tabular}

FIG. 6. Tip-sample distance dependence of amplitude of $2 \omega_{\mathrm{m}}$ component in the cantilever deflection signal measured when $f_{\mathrm{m}}$ was set at $10 \mathrm{kHz}$, which was well below $f_{1}$ in (a) $1 \mathrm{mM} \mathrm{KCl}$ aqueous solution, (b) acetonitrile, and (c) acetic acid. Those measured when $f_{\mathrm{m}}$ was set at the second resonance frequency in (d) $1 \mathrm{mM} \mathrm{KCl}$ aqueous solution, (e) acetonitrile, and (f) acetic acid are also shown. 
the increase in the capacitive force when the tip was brought closer to the surface in the measurements shown in Fig. 6. We divided the electrostatic force acting on the cantilever with a tip into three parts in Sec. IV; we take the voltage division along the electric line of flux into account. We calculate the electrostatic force acting on each part by integrating the force acting on the unit area. First, we calculate the capacitance per unit area of the cantilever $\left(\delta C_{\mathrm{B}_{\mathrm{cl}}}\right)$, the conical part of the tip $\left(\delta C_{\mathrm{B} \_ \text {cone }}\right)$, and the tip apex $\left(\delta C_{\mathrm{B} \_ \text {apex }}\right)$ (see Subsection 2 of Appendix). We consider that in polar liquid media, the double layer capacitance per unit area $\delta C_{\mathrm{EDL}}$ are connected in series with each $\delta C_{\mathrm{B}}$ at both ends of the corresponding line of electric flux, as shown in Fig. 3. Most of the voltage applied between the cantilever tip and sample surface is effectively applied on the smallest capacitance. Therefore, we compare the maximum value in $\delta C_{\mathrm{B}}$ of each part, which are given by the following equations:

$$
\begin{aligned}
\delta C_{\mathrm{B} \_ \text {apex }(\max )} & =\delta C_{\mathrm{B} \_ \text {apex }}(\theta=0), \\
\delta C_{\mathrm{B} \_ \text {cone }(\max )} & =\delta C_{\mathrm{B} \_ \text {cone }}\left(z^{\prime}=z_{\mathrm{B}}\right), \text { and } \\
\delta C_{\mathrm{B} \_\mathrm{cl}(\max )} & =\delta C_{\mathrm{B} \_\mathrm{cl}}\left(x=r_{0}\right) .
\end{aligned}
$$

Figure 7 compares the maximum value in $\delta C_{\mathrm{B}}$ of each part and $\delta C_{\mathrm{EDL}}$ in various liquid media. For the value of $\delta C_{\mathrm{EDL}}$ in each media, we used the Stern layer capacitance per area $\left(\delta C_{\mathrm{S}}\right)$ because of the following reasons.

Charge and discharge of the diffuse layer are accompanied with ionic current flow in the bulk solution. When the frequency of the applied alternating voltage is very high, the impedance of $C_{\mathrm{B}}$ becomes much smaller than $R_{\mathrm{B}}$ in parallel, and the diffuse layer does not respond to the applied alternating voltage. The threshold frequency, namely, the characteristic dielectric relaxation frequency of the diffuse layer is

(a)

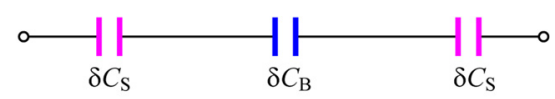

(b)

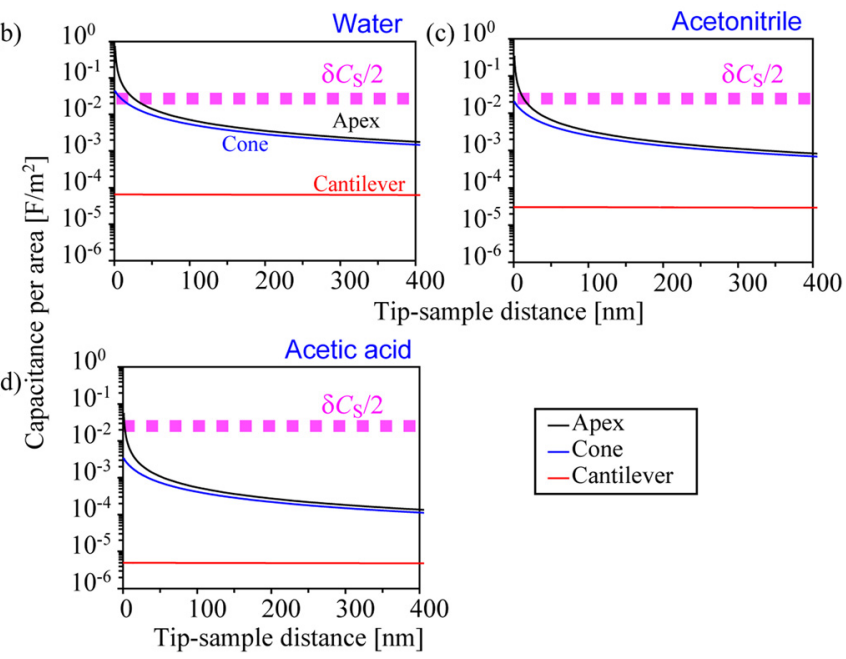

FIG. 7. (a) Equivalent circuit along each electric line of flux between a unit area on a cantilever with a tip and that on a sample surface. Calculated capacitance per unit area of tip apex, conical part of the tip, and cantilever in (b) water, (c) acetonitrile, and (d) acetic acid as a function of the tip-sample distance. The broken line in each figure shows a half the value of the Stern layer capacitance per unit area. expressed as the following equation (see Subsection 3 of Appendix):

$$
f_{\mathrm{c}}=\frac{1}{2 \pi \varepsilon_{0} \varepsilon_{\mathrm{r}} \rho} \propto \frac{n_{\infty}}{\varepsilon_{\mathrm{r}}} .
$$

When $f_{\mathrm{m}}$ is greater than $f_{\mathrm{c}}$, we can neglect $C_{\mathrm{D}}$, and only $C_{\mathrm{S}}$ and $C_{\mathrm{B}}$ have to be considered. For example, $f_{\mathrm{c}}$ of pure water, acetonitrile and acetic acid are much lower than tens of $\mathrm{kHz}$ as listed in Table I; therefore, $C_{\mathrm{D}}$ can be ignored in the case of these media. $C_{\mathrm{S}}$ can be measured by differential capacity curve, and the typical $C_{\mathrm{S}}$ of water is $0.2-0.3 \mathrm{~F} / \mathrm{m}^{2}{ }^{39}$ But the value of $C_{\mathrm{S}}$ observed in the electrostatic force measurement is in the range of $0.01-0.1 \mathrm{~F} / \mathrm{m}^{2}$ depending on the codition of surfaces because surface contamination may be contributed to the voltage division effect. Therefore, we used same value of $0.05 \mathrm{~F} / \mathrm{m}^{2}$ in all the media. Note that the EDL capacitance may not behave as an ideal capacitor rather the constant phase element due to the electrode surface roughness. ${ }^{39}$ However, in a high frequency range, we can treat $C_{\mathrm{S}}$ as an ideal capacitor because the charge transfer at the electrode interface does not occur.

We plotted $\delta C_{\mathrm{s}} / 2$ in Fig. 7 considering that the EDL exists on both surfaces and they are connected in series. In all liquid media, $\delta C_{\mathrm{s}} / 2$ is greater than $\delta C_{\mathrm{B} \_\mathrm{cl}(\max )}$; therefore, the electrostatic force acting on the cantilever is not affected. On the other hand, in acetonitrile and acetic acid, $\delta C_{\mathrm{B}_{\text {_cone(max) }}}$ is smaller than $\delta C_{\mathrm{s}} / 2$ by about a factor of 10 , which explains why we observed a slight increase in the capacitive force in Fig. 6. However, in all liquid media, $\delta C_{\mathrm{B} \_ \text {apex }}$ is always larger than $\delta C_{\mathrm{s}} / 2$, which implies that the capacitive force is not acting on the tip apex, and the amplitude of the capacitive force between the tip-sample does not contain any local information beneath the tip apex.

Next, we calculate the electrostatic forces acting on a cantilever with a tip considering the voltage drop at $C_{\mathrm{S}}$. The electrostatic force is not contributed by $C_{\mathrm{S}}$ because the relaxation frequency of Stern layer is much higher than the modulation frequency. Now the total electrostatic force contributed by $C_{\mathrm{B}}$ can be expressed as

$$
\begin{aligned}
F_{\text {esf }}= & F_{\text {apex }}+F_{\text {cone }}+F_{\text {cl }}=\frac{1}{2} V^{2}\left(\iint_{S} \frac{\partial \delta C_{\mathrm{B}_{-} \text {apex }}}{\partial z} \zeta_{\text {apex }}^{2} \mathrm{~d} S\right. \\
& \left.+\iint_{S} \frac{\partial \delta C_{\text {B_cone }}}{\partial z} \zeta_{\text {cone }}^{2} \mathrm{~d} S+\iint \frac{\phi_{1}(x)}{\phi_{1}(l)} \frac{\partial \delta C_{\text {B_cl }}}{\partial z} \zeta_{\text {cl }}^{2} \mathrm{~d} S\right),
\end{aligned}
$$

where $\xi_{\text {part }}$ is a voltage division ratio in the series circuit composed of $C_{\mathrm{B}}$ and two $C_{\mathrm{s}}$, which is used as a weight function for applied voltage. It is difficult to express $\xi_{\text {part }}$ as a simple equation especially when the electric double layers of a tip and sample surface are overlapped. However, we can neglect $R_{\mathrm{CT}}$ and $Z_{\mathrm{W}}$ when the modulation frequency is so high that the charge transfer and the mass transfer do not respond. In such a case, only the capacitances, $C_{\mathrm{S}}$ and $C_{\mathrm{B}}$ remain to be considered. Therefore, $\zeta_{\text {part }}$ can be expressed as a simple equation as 


$$
\lim _{\omega_{\mathrm{m}} \rightarrow \infty} \xi_{\text {part }}=\frac{\delta C_{\mathrm{S}} / 2}{\delta C_{\mathrm{S}} / 2+\delta C_{\text {B_part }}} .
$$

Figure 8(a) shows a theoretical voltage division ratio at the apex of the tip. Fig. 8 shows that only half of the applied alternating voltage is applied to $C_{\mathrm{B}_{-} \text {apex }}$ at the tip-sample distance of $20 \mathrm{~nm}$ in pure water. Figure 8(b) compares theoretically calculated $F_{\text {esf }}$ in non-polar media, water, acetonitrile, and acetic acid. Figure 8(c) shows those normalized at their values at the distance of $400 \mathrm{~nm}$. In non-polar media, $F_{\text {esf }}$ shows a steep increase as the tip is brought close to the sample surface. On the other hand, $F_{\text {esf }}$ in water and acetonitrile are strongly attenuated by the voltage drop at $C_{\mathrm{S}}$. The distance dependence of $F_{\text {esf }}$ in acetic acid is relatively similar to that in non-polar media. These calculations are consistent with the experimental results shown in Fig. 6, suggesting that the spatial resolution in the capacitive force measurement, i.e., the measurement of the dielectric properties at the solid/liquid interfaces, is strongly dependent on the polarization of the media.

To achieve a higher spatial resolution in the electrostatic force measurement, it is desirable that the electrostatic force shows a steep increase as a function of the tip-sample distance. The shorter the decay length of the electrostatic force $\left(\lambda_{\text {esf }}\right)$, the higher the achievable spatial resolution. $\lambda_{\text {esf }}$ is determined by the voltage division ratio $\xi_{\text {apex }}$, which is a function of the Stern layer capacitance $\left(C_{\mathrm{S}}\right)$ and the dielectric constant $\left(\varepsilon_{\mathrm{r}}\right)$ of the medium. In a liquid with a lower dielectric constant and a higher Stern layer capacitance, $\lambda_{\text {esf }}$ becomes shorter. We fitted the calculated curves in Fig. 8 to an exponential function as

$$
F_{\text {esf }}=F_{\text {esf_0 }} \exp \left(-z / \lambda_{\text {esf }}\right)+F_{\text {esf_o }},
$$

where $F_{\text {esf_0 }}$ and $F_{\text {esf_o }}$ are the fitting parameters. $\lambda_{\text {esf }}$ in water, acetonitrile, and acetic acid were determined as 200 , 60 , and $30 \mathrm{~nm}$, respectively. On the other hand, from the experimental results in Figs. 6(e) and 6(f), $\lambda_{\text {esf }}$ in acetonitrile and acetic acid were determined as 560 and $170 \mathrm{~nm}$, respectively. They are slightly longer than expected probably because the solvents used in the experiments contain some impurities and $f_{\mathrm{m}}$ used in the experiments were not high enough, which will be discussed in Sec. VI.

\section{CRITERIA FOR LOCAL CAPACITIVE FORCE DETECTION IN LIQUIDS}

As mentioned in the previous sections, it is difficult to detect local capacitive force acting on the tip apex by the conventional voltage modulation techniques. In this section, we discuss the criteria for local capacitive force measurements in polar liquid media.

First, the experimental results in Fig. 4 showed that the cantilever deflection is caused by not only by the electrostatic force but also by the surface stress. The equivalent circuit of the medium between the cantilever and sample surface are different depending on the modulation frequency $\left(f_{\mathrm{m}}\right)$, as shown in Fig. 9(a). The surface stress rolls off at $f_{\text {ss_c }}$, which is equal to $f_{\mathrm{c}}$ in the case of weak electrolyte (see (a)

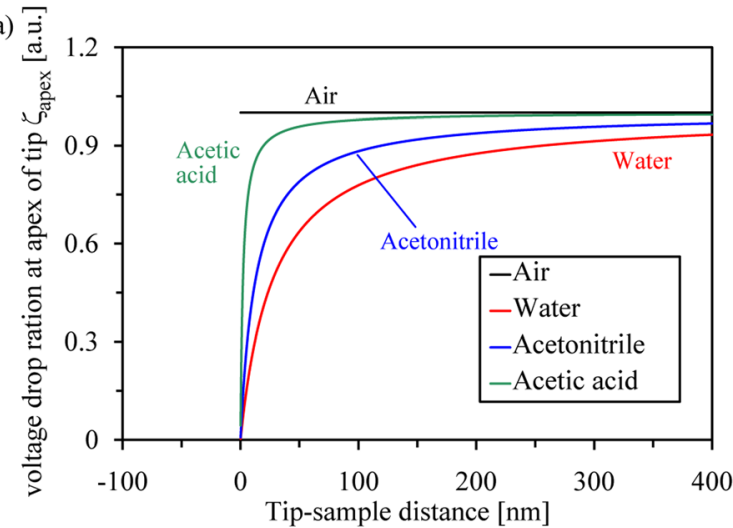

(b)

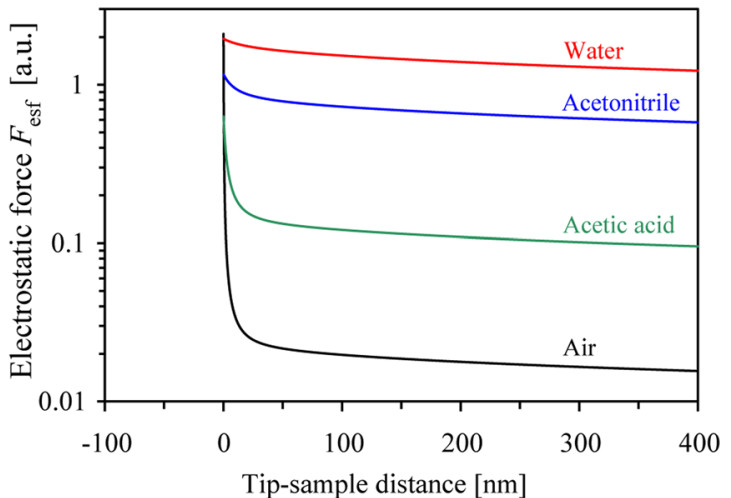

(c)

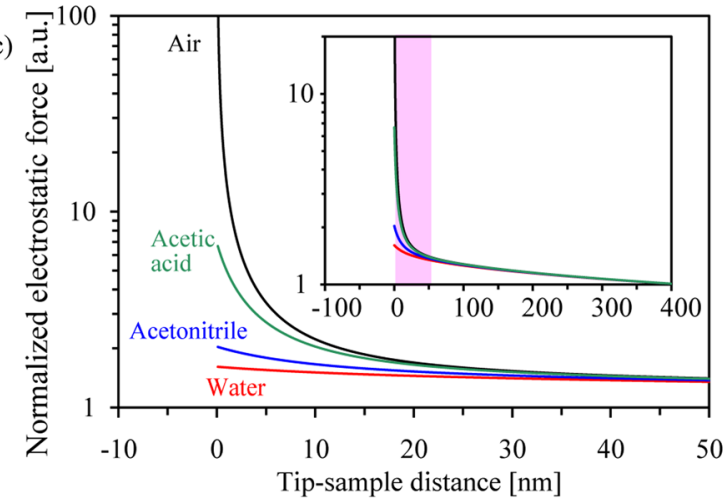

FIG. 8. (a) Calculated voltage division ratio on capacitance per unit area at tip apex as a function of the tip-sample distance. (b) Calculated electrostatic force as a function of the tip-sample distance in non-polar media, pure water, acetonitrile, and acetic acid. (c) Calculated electrostatic force normalized at the distance of $400 \mathrm{~nm}$.

Subsection 3 of Appendix). The surface stress on the cantilever surface is dominant over that on the tip surface because of the larger surface area of the cantilever in the upper parts of Figs. 9(b) and 9(c). Since the surface stress contribution to the cantilever deflection is dominant up to the threshold frequency $f_{\mathrm{t}}$, we should choose $f_{\mathrm{m}}$ at a frequency much higher than $f_{\mathrm{t}}$, as shown in Fig. 9(c). Thus, the first criterion for $f_{\mathrm{m}}$ can be expressed as

$$
f_{\mathrm{m}} \gg f_{\mathrm{t}}
$$

The electrostatic force acting on the cantilever $\left(F_{\mathrm{cl}}\right)$ is only slightly increased when the tip is brought close to the sample surface because of the tip height, as shown in Fig. 9(c). It is important for local capacitive force measurements that the electrostatic force acting on the tip $\left(F_{\text {apex }}\right)$ is increased when 


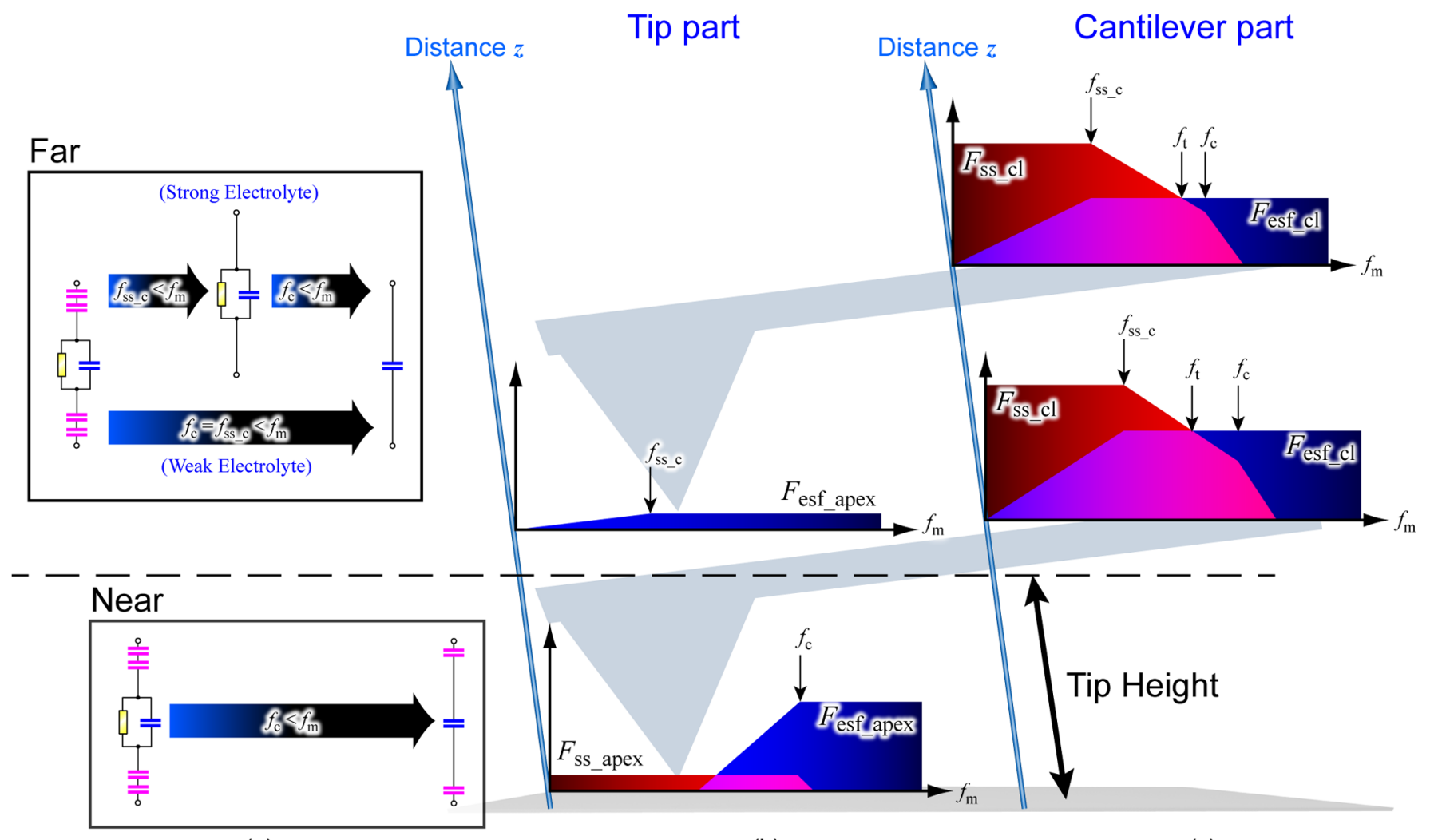

(a)

(b)

(c)

FIG. 9. Visual summary of criteria for electrostatic force detection induced by applying an alternating voltage between a cantilever with a tip and a sample surface in polar liquid media. The equivalent circuits of the medium are different depending on the modulation frequency $\left(f_{\mathrm{m}}\right)$ and the tip-sample distance, as shown in (a). Frequency spectra of the electrostatic force acting on the tip part and the cantilever part, which are different depending on the tip-sample distance, are schematically shown in (b) and (c), respectively.

the tip is brought close to the surface. ${ }^{41}$ From the discussion in Secs. III and IV, $F_{\text {apex }}$ shows an increase only when $f_{\mathrm{m}}$ is greater than $f_{\mathrm{c}}$, at which $C_{\mathrm{D}}$ and other electrochemical components can be ignored, as shown in the lower parts of Figs. 9(a) and 9(b). Therefore, the second criterion for $f_{\mathrm{m}}$ can be expressed as

$$
f_{\mathrm{m}} \gg f_{\mathrm{c}} \text {. }
$$

In a liquid with a higher resistivity, these criteria are easily met (see Subsection 3 of Appendix). Since the cantilever should response to the electrostatic force at $2 f_{\mathrm{m}}$, the cantilever with a resonance frequency higher than $2 f_{\mathrm{m}}$ should be used or $2 f_{\mathrm{m}}$ should be tuned at one of the resonance frequencies of the cantilever. Although the capacitive force measurement can be conducted with a cantilever of relatively low resonance frequency by the use of the heterodyne techniques, ${ }^{41,42}$ essential spatial resolution is limited by the dielectric constant of medium. Therefore, we consider that we should use a modulation frequency even higher than the relaxation frequency of the polar molecules in the solvent (in the case of water, more than tens of $\mathrm{GHz}$ for instance), where the voltage division ratio $\xi$ is considered to be 1 and the voltage drop at the Stern layer becomes negligibly small. In such a case, it might be possible to perform local dielectric constant measurements with a spatial resolution, which is same as that in air or vacuum environment.

\section{CONCLUSIONS}

We measured the capacitive force induced on a conductive cantilever with a tip when an alternating voltage is applied between the cantilever and a sample surface in various liquids including polar liquid media. We found the cantilever deflection is predominantly caused by the surface stress in polar liquid media especially when the modulation frequency is low, and that the electrostatic force contribution on the cantilever deflection becomes dominant in a high frequency range which is typically higher than tens of $\mathrm{kHz}$. In polar liquid media, as the tip-sample distance is decreased, the bulk solution capacitance is increased and more part of the applied alternating voltage drops on the EDLs, which makes the electrostatic force contribution smaller. Furthermore, when the tip-sample distance is reduced to less than $100 \mathrm{~nm}$, the capacitance of the Stern layer per unit area becomes comparable to the capacitance per unit area on the cantilever tip apex. The capacitive force is no longer acting on the tip apex, and the amplitude of the capacitive force between the tip-sample does not include local information beneath the tip apex.

We proposed criteria for local capacitive force measurements in polar liquid media. First, the modulation frequency must be much higher than the characteristic dielectric relaxation frequency as well as the transition frequency at which the electrostatic force contribution originating from the capacitance between the tip and sample becomes predominant. The capacitance per unit area of the tip apex and sample 
surface must be much lower than the Stern layer capacitance per area. Although local capacitive force measurement becomes possible if these criteria are fulfilled, its spatial resolution is much less than that in air or vacuum environment without the modulation frequency even higher than the relaxation frequency of the polar molecules in the solvent is used.

\section{ACKNOWLEDGMENTS}

This work was supported by a Grant-in-Aid for Scientific Research from the Ministry of Education, Culture, Sports, Science and Technology of Japan, SENTAN Program of the Japan Science and Technology Agency. The authors would also like to thank Ryohei Kokawa, Masahiro Ohta, and Kazuyuki Watanabe of Shimadzu Corporation.

\section{APPENDIX: THEORETICAL CALCULATIONS}

\section{Calculation of electrostatic force acting on a cantilever with a tip}

The electrostatic force acting on a cantilever with a tip is considered as a sum of the electrostatic forces acting on tip apex, cone, and cantilever parts. $F_{\text {apex }}$ and $F_{\text {cone }}$ are given in Ref. 34 as

$$
F_{\text {apex }}=-\pi \varepsilon_{0} \varepsilon_{\mathrm{r}} R_{\text {apex }}^{2} \frac{1-\sin \theta_{\text {cone }}}{z\left[z+R_{\text {apex }}\left(1-\sin \theta_{\text {cone }}\right)\right]} V^{2}
$$

and

$$
F_{\text {cone }}=-\frac{\pi \varepsilon_{0} \varepsilon_{\mathrm{r}} V^{2}}{\left[\ln \tan \left(\theta_{\text {cone }} / 2\right)\right]^{2}}\left[\ln \left(\frac{z_{\mathrm{A}}}{z_{\mathrm{B}}}\right)+z_{\mathrm{C}}\left(\frac{1}{z_{\mathrm{A}}}-\frac{1}{z_{\mathrm{B}}}\right)\right],
$$

respectively, where $\varepsilon_{0}$ and $\varepsilon_{\mathrm{r}}$ are the vacuum permittivity and relative dielectric constant, $R_{\text {apex }}$ and $\theta_{\text {cone }}$ are the tip radius and the half cone angle of the tip, respectively see Fig. 10 for the definition of variables. $z_{\mathrm{A}}, z_{\mathrm{B}}$, and $z_{\mathrm{C}}$ are given by

$$
\begin{aligned}
& z_{\mathrm{A}}=z+h, \\
& z_{\mathrm{B}}=z+R_{\text {apex }}\left(1-\sin \theta_{\text {cone }}\right), \text { and } \\
& z_{\mathrm{C}}=z+R_{\text {apex }}-\frac{R_{\text {apex }}}{\tan \theta_{\text {cone }}},
\end{aligned}
$$

respectively, ${ }^{34}$ where $h$ is the tip height.

\section{Calculation of capacitance per unit area of each part of cantilever}

The electrostatic forces acting on the tip apex and the conical part of the tip were calculated by integrating the differential capacitance per area following Ref. 34 as

$$
\frac{\partial C_{\text {apex }}(z)}{\partial z}=\int_{0}^{\pi / 2-\theta_{\text {cone }}}\left[-2 \pi \varepsilon_{0} \varepsilon_{\mathrm{r}} \frac{\sin ^{2} \theta}{\theta\left(z / R_{\text {apex }}+1-\cos \theta\right)^{2}}\right] \mathrm{d} \theta
$$

and

$$
\frac{\partial C_{\text {cone }}(\mathrm{z})}{\partial z}=\int_{z_{\mathrm{B}}}^{z_{\mathrm{A}}}\left\{-\frac{2 \pi \varepsilon_{0} \varepsilon_{\mathrm{r}}}{\left[\ln \tan \left(\theta_{\text {cone }} / 2\right)\right]^{2}} \frac{z^{\prime}-z_{\mathrm{C}}}{z^{\prime 2}} \mathrm{~d} z^{\prime}\right\},
$$

respectively. In a similar way, the differential capacitance of the cantilever is given by

$$
\frac{\partial C_{\mathrm{cl}}(\mathrm{z})}{\partial z}=\int_{r_{0}}^{r_{0}+l}\left[-\varepsilon_{0} \varepsilon_{\mathrm{r}} w \frac{\cot \left(\theta_{\mathrm{cl}} / 2\right)}{2 \theta_{\mathrm{cl}}} \frac{1}{x^{2}}\right] \mathrm{d} x
$$

after Ref. 35. The capacitance of the tip apex, the conical part of the tip, and the cantilever can be obtained by integrating the above equations with respect to the tip-sample distance $(z)$ as

$$
\begin{gathered}
C_{\mathrm{B}_{-} \text {apex }}(z)=\int_{0}^{\pi / 2-\theta_{\text {cone }}} 2 \pi \varepsilon_{0} \varepsilon_{\mathrm{r}} R_{\text {apex }} \frac{\sin ^{2} \theta}{\theta\left(z / R_{\text {apex }}+1-\cos \theta\right)} \mathrm{d} \theta, \\
C_{\text {B_cone }}(z)=\int_{z_{\mathrm{B}}}^{z_{\mathrm{A}}} \frac{2 \pi \varepsilon_{0} \varepsilon_{\mathrm{r}}}{\left[\ln \tan \left(\theta_{\text {cone }} / 2\right)\right]^{2}} \frac{z^{\prime}-z_{\mathrm{C}}}{z^{\prime}} \mathrm{d} z^{\prime}, \text { and } \\
C_{\mathrm{B}_{-} \mathrm{cl}}(z)=\int_{r_{0}}^{r_{0}+l} \varepsilon_{0} \varepsilon_{\mathrm{r}} \frac{w}{\theta_{\mathrm{cl}}} \frac{1}{x} \mathrm{~d} x,
\end{gathered}
$$

respectively. On the other hand, the surface areas of the apex, cone and cantilever parts are given by integrating infinitesimal area elements as

$$
\begin{gathered}
S_{\text {apex }}=\int_{0}^{\pi / 2-\theta_{\text {cone }}} 2 \pi R_{\text {apex }}^{2} \sin \theta \mathrm{d} \theta, \\
S_{\text {cone }}=\int_{z_{\mathrm{B}}}^{z_{\mathrm{A}}} \frac{2 \pi \sin \theta_{\text {cone }}}{\cos ^{2} \theta_{\text {cone }}}\left(z^{\prime}-z_{\mathrm{C}}\right) \mathrm{d} z^{\prime}, \quad \text { and } \\
S_{\mathrm{cl}}=\int_{r_{0}}^{r_{0}+l} w \mathrm{~d} x,
\end{gathered}
$$

respectively. The capacitance per unit area can be obtained by dividing the integrand of the capacitance by infinitesimal area element as

$$
\begin{gathered}
\delta C_{\text {B_apex }}\left(0 \leq \theta \leq \frac{\pi}{2}-\theta_{\text {cone }}\right)=\frac{\varepsilon_{0} \varepsilon_{\mathrm{r}}}{R_{\text {apex }}} \frac{\sin \theta}{\theta\left(z / R_{\text {apex }}+1-\cos \theta\right)}, \\
\delta C_{\text {B_cone }}\left(z_{\mathrm{B}} \leq z^{\prime} \leq z_{\mathrm{A}}\right)=\frac{\varepsilon_{0} \varepsilon_{\mathrm{r}}}{\left[\ln \tan \left(\theta_{\text {cone }} / 2\right)\right]^{2}} \frac{\cos ^{2} \theta_{\text {cone }}}{\sin \theta_{\text {cone }}} \frac{1}{z^{\prime}},
\end{gathered}
$$

and

$$
\delta C_{\mathrm{B} \_\mathrm{cl}}\left(r_{0} \leq x \leq r_{0}+l\right)=\frac{\varepsilon_{0} \varepsilon_{\mathrm{r}}}{\theta_{\mathrm{cl}}} \frac{1}{x},
$$

respectively. 
(a)

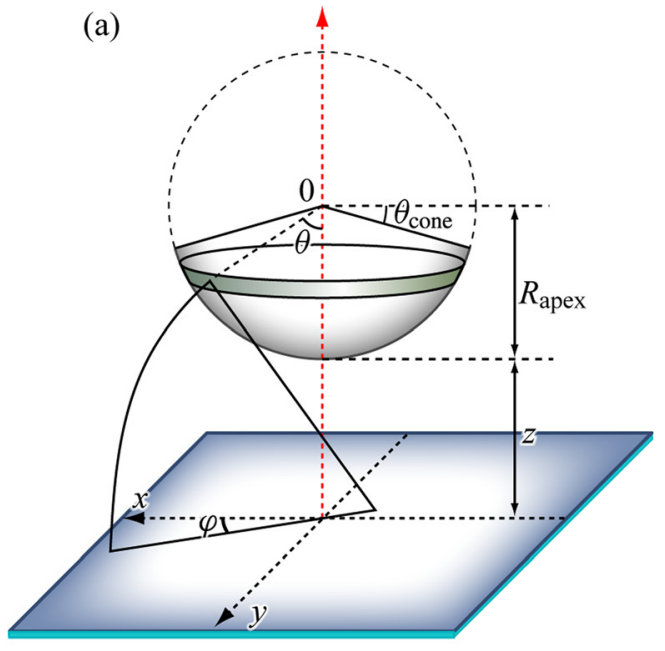

(b)

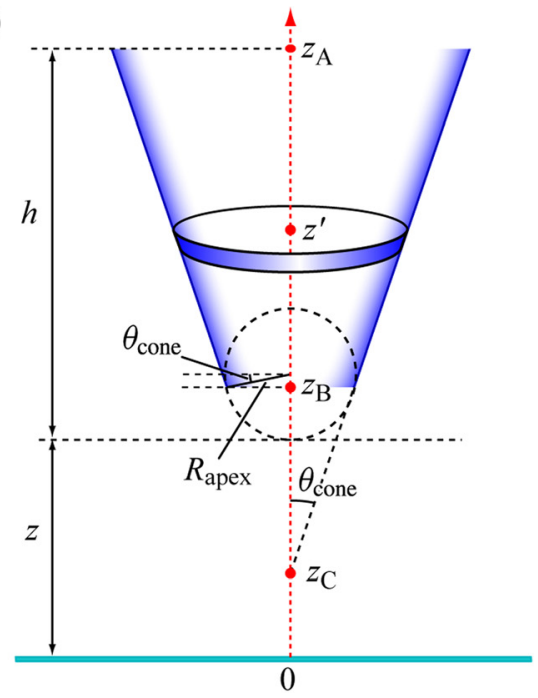

FIG. 10. Models for calculation of the electrostatic forces acting on (a) apex, (b) cone, and (c) cantilever parts.

(c)

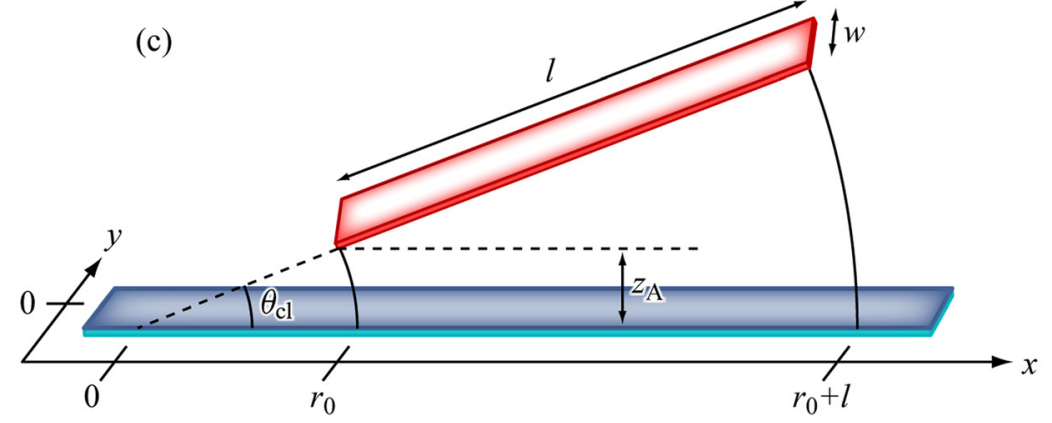

\section{Cutoff frequency of surface stress contribution}

The characteristic dielectric relaxation frequency of an electrolyte, which is calculated from the resistivity and dielectric constant as

$$
f_{\mathrm{c}}=\frac{1}{2 \pi R_{\mathrm{B}} C_{\mathrm{B}}}=\frac{1}{2 \pi \varepsilon_{0} \varepsilon_{\mathrm{r}} \rho} \propto n_{\infty}
$$

where $\rho$ is the resistivity of the electrolyte, and $n_{\infty}$ is the number of ions per unit volume. Therefore, $f_{\mathrm{c}}$ is proportional to the concentration of the electrolyte.

On the other hand, the cutoff frequency of the surface stress contribution is equal to the cutoff frequency of the $R C$ filter composed of the bulk-solution resistance and the two electric double layer capacitances in ideal conditions, and it is given by

$$
\begin{aligned}
f_{\text {ss } \_\mathrm{c}} & =\frac{1}{2 \pi R_{\mathrm{B}}\left(C_{\mathrm{EDL}} / 2\right)}=\frac{1}{\pi \kappa_{\mathrm{D}} \varepsilon_{0} \varepsilon_{\mathrm{r}} \rho_{\mathrm{B}}(z+h)} \\
& =\frac{2 f_{\mathrm{c}}}{\kappa_{\mathrm{D}}(z+h)} \propto n_{\infty}^{1 / 2},
\end{aligned}
$$

where $\kappa_{\mathrm{D}}$ is the inverse Debye length. Therefore, $f_{\text {ss_c }}$ is proportional to the square root of the concentration of the electrolyte. In the case of weak electrolyte, $f_{\text {ss_c }}$ is higher than the $f_{\mathrm{c}}$, and hence the surface stress contribution rolls off at $f_{\mathrm{c}}$.

${ }^{1}$ T. Fukuma, K. Kobayashi, K. Matsushige, and H. Yamada, Appl. Phys. Lett. 87, 034101 (2005).
${ }^{2}$ B. W. Hoogenboom, H. J. Hug, Y. Pellmont, S. Martin, P. L. T. M. Frederix, D. Fotiadis, and A. Engel, Appl. Phys. Lett. 88, 193109 (2006).

${ }^{3}$ T. Ando, T. Uchihashi, and T. Fukuma, Prog. Surf. Sci. 83, 337 (2008).

${ }^{4}$ H. Yamada, K. Kobayashi, T. Fukuma, Y. Hirata, T. Kajita, and K. Matsushige, Appl. Phys. Express 2, 095007 (2009).

${ }^{5}$ K.-I. Umeda and K.-I. Fukui, J. Vac. Sci. Technol. B 28, C4D40 (2010).

${ }^{6}$ K.-I. Umeda and K.-I. Fukui, Langmuir 26, 9104 (2010).

${ }^{7}$ B. P. Lynch, A. M. Hilton, C. H. Doerge, and G. J. Simpson, Langmuir 21, 1436 (2005).

${ }^{8}$ L. Fumagalli, G. Ferrari, M. Sampietro, and G. Gomila, Nano Lett. 9, 1604 (2009)

${ }^{9}$ Y. Martin, D. W. Abraham, and H. K. Wickramasinghe, Appl. Phys. Lett. 52, 1103 (1988)

${ }^{10}$ J. E. Stern, B. D. Terris, H. J. Mamin, and D. Rugar, Appl. Phys. Lett. 53, 2717 (1988)

${ }^{11}$ J. R. Matey and J. Blanc, J. Appl. Phys. 57, 1437 (1985).

${ }^{12}$ C. D. Bugg and P. J. King, J. Phys. E: Sci. Instrum. 21, 147 (1988).

${ }^{13}$ C. C. Williams, W. P. Hough, and S. A. Rishton, Appl. Phys. Lett. 55, 203 (1989).

${ }^{14}$ R. Shao, S. V. Kalinin, and D. A. Bonnell, Appl. Phys. Lett. 82, 1869 (2003).

${ }^{15}$ K. J. Lai, H. L. Peng, W. Kundhikanjana, D. T. Schoen, C. Xie, S. Meister, Y. Cui, M. A. Kelly, and Z. X. Shen, Nano Lett. 9, 1265 (2009).

${ }^{16}$ W. Lu, D. Wang, and L. W. Chen, Nano Lett. 7, 2729 (2007).

${ }^{17}$ G. Gramse, I. Casuso, J. Toset, L. Fumagalli, and G. Gomila, Nanotechnology 20, 395702 (2009).

${ }^{18}$ C. Riedel, R. Arinero, P. Tordjeman, M. Ramonda, G. Leveque, G. A. Schwartz, D. G. de Oteyza, A. Alegria, and J. Colmenero, J. Appl. Phys. 106, 024315 (2009).

${ }^{19}$ L. Fumagalli, G. Gramse, D. Esteban-Ferrer, M. A. Edwards, and G. Gomila, Appl. Phys. Lett. 96, 183107 (2010).

${ }^{20}$ K. Kobayashi, H. Yamada, and K. Matsushige, Appl. Phys. Lett. 81, 2629 (2002).

${ }^{21}$ Y. Hirata, F. Mizutani, and H. Yokoyama, Surf. Interface Anal. 27, 317 (1999).

${ }^{22}$ M. P. Scherer, G. Frank, and A. W. Gummer, J. Appl. Phys. 88, 2912 (2000). 
${ }^{23}$ B. J. Rodriguez, S. Jesse, K. Seal, A. P. Baddorf, and S. V. Kalinin, J. Appl. Phys. 103, 014306 (2008).

${ }^{24}$ K.-I. Umeda, N. Oyabu, K. Kobayashi, Y. Hirata, K. Matsushige, and H. Yamada, Appl. Phys. Express 3, 065205 (2010).

${ }^{25}$ K.-I. Umeda, K. Kobayashi, K. Matsushige, and H. Yamada, Appl. Phys. Lett. 101, 123112 (2012).

${ }^{26}$ N. Kobayashi, H. Asakawa, and T. Fukuma, Rev. Sci. Instrum. 81, 123705 (2010).

${ }^{27}$ K.-I. Umeda, K. Kobayashi, N. Oyabu, Y. Hirata, K. Matsushige, and H. Yamada, "Practical aspects of Kelvin-probe Force Microscopy at solid/ liquid interfaces in various liquid media" (unpublished).

${ }^{28}$ J. E. Sader, J. W. M. Chon, and P. Mulvaney, Rev. Sci. Instrum. 70, 3967 (1999).

${ }^{29} \mathrm{FC}-70$ datasheet is available at http://solutions.3m.com/wps/portal/3M/ en_US/ElectronicsChemicals/Home/Products/ElectronicLiquids/ (Accessed May, 2012).

${ }^{30}$ G. C. Benson and A. R. Gordon, J. Chem. Phys. 13, 473 (1945).

${ }^{31}$ T. D. Sawyer, A. Sobkowiak, and J. L. Roberts, Jr., Electrochemistry for Chemists, 2nd ed. (John Wiley \& Sons, Inc., 1995).
${ }^{32}$ M. Nonnenmacher, M. P. O'Boyle, and H. K. Wickramasinghe, Appl. Phys. Lett. 58, 2921 (1991).

${ }^{33}$ J. M. R. Weaver and D. W. Abraham, J. Vac. Sci. Technol. B 9, 1559 (1991).

${ }^{34}$ S. Hudlet, M. Saint Jean, C. Guthmann, and J. Berger, Eur. Phys. J. B 2, 5 (1998).

${ }^{35}$ J. Colchero, A. Gil, and A. M. Baró, Phys. Rev. B 64, 245403 (2001).

${ }^{36}$ M. V. Salapaka, H. S. Bergh, J. Lai, A. Majumdar, and E. McFarland, J. Appl. Phys. 81, 2480 (1997).

${ }^{37}$ J. E. Sader, J. Appl. Phys. 84, 64 (1998).

${ }^{38}$ R. Raiteri and H.-J. Butt, J. Phys. Chem. 99, 15728 (1995).

${ }^{39}$ A. J. Bard and L. R. Faulkner, Electrochemical methods, Fundamentals and Applications, 2nd ed. (John Wiley \& Sons, Inc., 2001).

${ }^{40}$ A. Kikukawa, S. Hosaka, and R. Imura, Appl. Phys. Lett. 66, 3510 (1995).

${ }^{41}$ We succeeded in local capacitive force measurement by the use of an amplitude-modulated high-frequency electric field, which will be published elsewhere.

${ }^{42}$ H. Yokoyama, M. J. Jeffery, and T. Inoue, Jpn. J. Appl. Phys. 32, L1845 (1993). 